\title{
Enhancement of flow mixing in micro and nano channels
}

\author{
A. K. Nayak ${ }^{1}$
}

(Received 18 December 2013; revised 13 March 2014)

\begin{abstract}
The aim is to increase the mixing potential of ion species in micro and nano channels with heterogeneous surface potential. We discuss the generation of vortical flow, due to the presence of wall heterogeneity at different locations in the channel. The flow characteristics are obtained by numerical solution of the Poisson equation, the Nernst-Planck equation, and the Navier-Stokes equation, simultaneously. A numerical method based on the pressure correction iterative algorithm is adopted to compute the flow field and mole fraction of the ions. The strong recirculation vortices which appear above the heterogeneities generate a strong pressure gradient which increases mixing performance.
\end{abstract}

http://journal.austms.org.au/ojs/index.php/ANZIAMJ/article/view/7814 gives this article, (C) Austral. Mathematical Soc. 2014. Published April 16, 2014, as part of the Proceedings of the 11th Biennial Engineering Mathematics and Applications Conference. ISSN 1446-8735. (Print two pages per sheet of paper.) Copies of this article must not be made otherwise available on the internet; instead link directly to this URL for this article. 


\section{Contents}

1 Introduction

C48

2 Formulation

C51

3 Results and discussions

C55

References

C62

\section{Introduction}

Electroosmotic flow (EOF) is the resulting fluid motion when an externally applied electric field interacts with charged ions in the diffused part of an electric double layer (EDL). This type of flow is observed in micro channel network devices for chemistry and biochemistry applications [1]. The mixing in channels with heterogeneous surface potential depends on the strength of the vortices induced by the potential patches. The EOF is proportional to the wall $\zeta$ potential as well as the applied external electric field. In microfluidic devices, electroosmosis is often utilized for simultaneous fluid transport and mixing. The Reynolds number is rarely effective to drive the charged ions in the case of EOF.

In general, passive and active mixers are used to enhance mixing inside the micro channel. Passive mixers use particular channel geometry configurations to increase the interfacial area between the liquids to be mixed [2]. The major difficulty in passive mixing is the fabrication complexity in the micro-scale. Active mixers introduce moving parts inside micro channels or apply an alternating electric field. Although these two mixing techniques effectively reduce the mixing time, they are difficult to integrate into microfluidic systems since they require external variable frequency pumps or internal mechanical moving parts [3]. Bhattacharyya and Nayak [4] provide a complete discussion 
of electroosmotic flow in micro and nano channels and the flow enhancement obtained from potential patches and blocks.

A micro channel with nonuniform $\zeta$ potential induces strong vortices in the EOF $[5,6]$. Erickson and $\mathrm{Li}[6]$ found that the re-circulation is strongest when the $\zeta$ potential of the heterogeneous surface is of equal and opposite sign to that of the homogeneous surface. Fu et al. [7] observed that a step change in $\zeta$ potential causes a significant variation in the velocity profile and pressure distribution. Watzig et al. [8] conducted an experimental study of surface roughness in both electrophoresis and electroosmosis in micro and nanochannels. Micro and nanofluidic channels have surface roughness of a few angstroms to a few micrometers. It is possible to achieve atomic flatness on the inner surfaces.

Major studies of EOF in homogeneous and heterogeneous channel are based on the Boltzmann distribution of ions, which leads to the Poisson-Boltzmann $(\mathrm{PB})$ equation for electric potential. Although the computational cost of the numerical computation of the exact Nernst-Plank (NP) model is high compare to that of the simple PB model, the advantages of the NP model overshadow this weakness. When the channel height is comparable to the EDL thickness, the core of the channel is no longer electrically neutral and hence the validity of the PB equation is questionable. Additionally, the Boltzmann distribution neglects the convection effect and the external electric field. For EOF near a steep jump in surface potential or close to surface roughness, the convection effect may not be negligible.

One of the objectives of the present study is to investigate the flow characteristics due to NP model heterogeneity in wall potential. To validate our results (NP model) with the PB model (i.e., symmetric boundary condition at the center line) one steady EOF comparison was calculated, since the NP model does not require the centerline symmetry condition or the low surface potential restriction.

In this article, the EOF in a nano mixing channel is considered by placing several overpotential patches at different locations on the channel walls, 

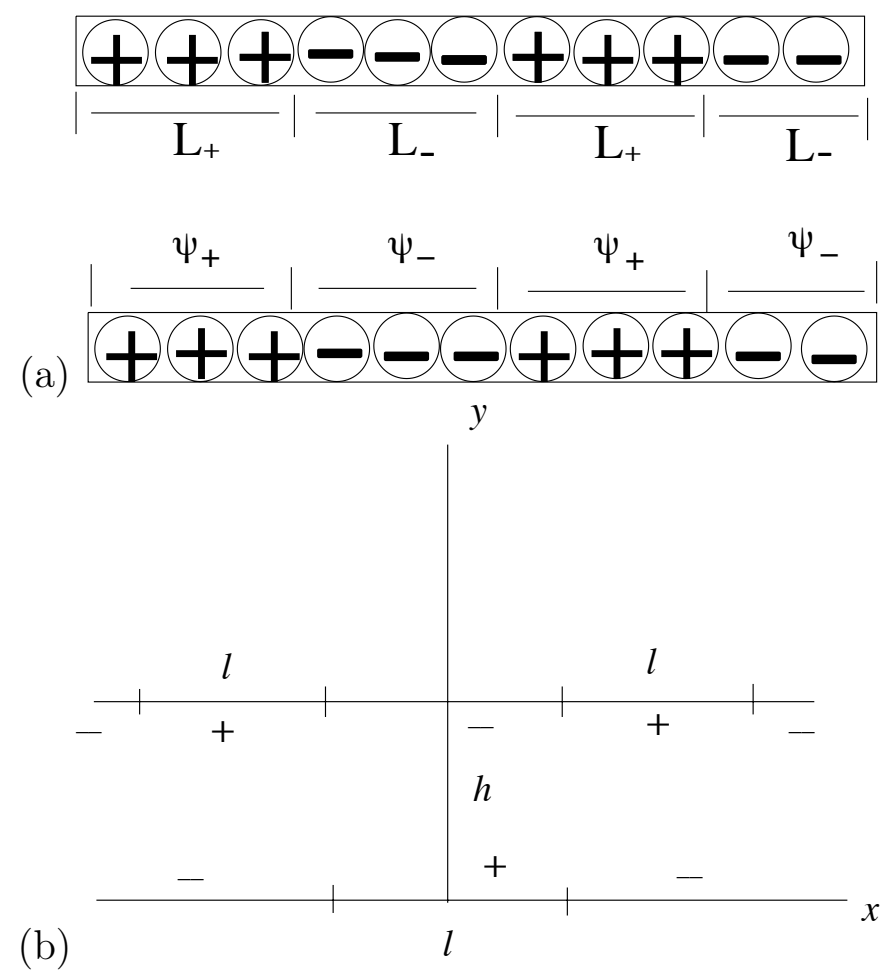

Figure 1: Schematic diagram of the nano channel and computational domain: (a) symmetric; (b) asymmetric.

where the patch length is of the order of the channel height. Two different configurations of the overpotential patches on the channel walls are considered.

(I) Four overpotential patches are placed symmetrically along the channel walls, that is, overpotential patches of equal length are placed on opposite sides of the channel at channel heights $y=0$ and $y=1$ (Figure 1(a)).

(II) Three overpotentials are placed asymmetrically, that is, between two overpotential patches in the lower wall, $y=0$, one overpotential patch is placed in the upper wall, $y=1$ (Figure 1(b)). 


\section{Formulation}

For the flow configuration we consider a long rectangular channel of nano constrained height. The channel is filled with an incompressible Newtonian electrolyte of uniform permittivity $\epsilon_{\mathrm{e}}$ and viscosity $\mu$ under the influence of an external electric field along the length of the channel, say $x$-axis. The surface heterogeneity is created by a stepwise surface potential, as shown in Figure 1(a). In Figure 1(a) the plus and minus patterns illustrate the symmetrical heterogeneous surface with equipotential surfaces of length $\mathrm{L}_{+}$and $\mathrm{L}_{-}$, positioned on the lower and upper channel walls. The $\mathrm{L}_{+}$and $\mathrm{L}_{-}$regions have potentials $\psi_{+}$and $\psi_{-}$, respectively. For computational purposes we considered a channel of height $h$ with patches (corresponding to $\psi_{+}$regions) of length $l \sim \mathcal{O}(h)$ (see Figure 1(b) for the asymmetric configuration). We assume that the electrodes are placed at the inlet and outlets of the channel and there is a constant imposed electric field in the $x$ direction.

The dimensional electric field $E^{*}=\left(E_{x}, E_{y}, E_{z}\right)$ satisfying Maxwell's equation, $\mathrm{E}^{*}=-\nabla \Phi^{*}$, is governed by

$$
\nabla \cdot\left(\epsilon_{e} \mathrm{E}^{*}\right)=\rho_{e}=\mathrm{F} \sum_{i} z_{i} c_{i}=F c \sum_{i} z_{i} X_{i},
$$

where $\rho_{e}$ is the charge density per unit volume, $\Phi^{*}$ the electric potential, $F$ is the Faraday's constant, $c_{i}$ is the molarity of ith ion species and $c$ is the total molar concentration which is assumed to be a constant. Here $X_{i}=c_{i} / c$ and $z_{\mathrm{i}}$ is the valence of species $i$. The superscript $*$ denotes a dimensional quantity.

The electric potential $\Phi^{*}(x, y, z)=-E_{0} x+\phi^{*}(x, y, z)$. Here, $E_{0}$ is the externally applied electric field, which is constant, and $\phi^{*}$ is the induced electric potential. Using equation (1), the nondimensional form of the perturbation potential for monovalent ions is

$$
\frac{\partial^{2} \phi}{\partial y^{2}}+\epsilon_{1}^{2} \frac{\partial^{2} \phi}{\partial x^{2}}+\epsilon_{2}^{2} \frac{\partial^{2} \phi}{\partial z^{2}}=-\frac{F c h^{2}}{\epsilon_{e} \phi_{0}} \sum_{i} z_{i} X_{i}
$$


The potential is scaled by $\phi_{0}=\mathrm{RT} / \mathrm{F}$ where $\mathrm{R}$ is the gas constant and $\mathrm{T}$ is the temperature of the liquid. The width of the channel is $W$ and for nondimensionalization we use $(l, h, W)$ for coordinates $(x, y, z)$, for example, $x=x^{*} / \mathrm{l}$. We define $\epsilon_{1}=\mathrm{h} / \mathrm{l}$ and $\epsilon_{2}=\mathrm{h} / \mathrm{W}$.

We assume the electrolyte consists of sodium ions and chlorine ions, with $\mathrm{Na}^{+}$ and $\mathrm{Cl}^{-}$the number of moles of sodium ions and chlorine ions, respectively. So, $\sum_{i} z_{i} X_{i}=X_{+}-X_{-}=g-f$, where $X_{+}=g=\mathrm{Na}^{+} / c$ is the mole fraction of the sodium cations and $X_{-}=f=\mathrm{Cl}^{-} / \mathrm{c}$ is the mole fraction of the chlorine anions.

The fluid and transport properties are assumed to be constants. The molar flux of species $i$ oriented perpendicularly to the flow is

$$
n_{i}=-D_{i} \nabla c_{i}+c_{i} \omega_{i} z_{i} F E+c_{i} \mathbf{u}^{*}
$$

Here $\mathbf{u}^{*}(u, v, w)$ is the velocity field of the fluid and $D_{i}$ is the diffusion coefficient of the ith species. The mobility $\omega_{i}$ is defined by the Nernst-Planck equation $\omega_{i}=D_{i} / R T$. The transport equation for species $i$ in steady EOF is governed by

$$
\nabla \cdot \mathfrak{n}_{\mathrm{i}}=0 .
$$

Using equations (3) and (4), the concentration equation for molar flux in nondimensional form is

$$
\begin{aligned}
\frac{\partial^{2} X_{i}}{\partial y^{2}}+\epsilon_{1}^{2} \frac{\partial^{2} X_{i}}{\partial x^{2}}+\epsilon_{2}^{2} \frac{\partial^{2} X_{i}}{\partial z^{2}}= & \operatorname{Pe}\left(\epsilon_{1} u \frac{\partial X_{i}}{\partial x}+v \frac{\partial X_{i}}{\partial y}+\epsilon_{2} w \frac{\partial X_{i}}{\partial z}\right) \\
& +\left(\epsilon_{1} z_{i} \frac{\partial X_{i} E_{x}}{\partial x}+z_{i} \frac{\partial X_{i} E_{y}}{\partial y}+\epsilon_{2} z_{i} \frac{\partial X_{i} E_{z}}{\partial z}\right)
\end{aligned}
$$

The nondimensional electric field components are $E_{x}=\frac{h E_{0}}{\phi_{0}}-\epsilon_{1} \frac{\partial \phi}{\partial x}, E_{y}=-\frac{\partial \phi}{\partial y}$ and $E_{z}=-\epsilon_{2} \frac{\partial \phi}{\partial z}$. The velocity components are scaled by the electroosmotic velocity $\mathrm{U}_{0}$, where $\mathrm{U}_{0}=\epsilon_{e} \mathrm{E}_{0} \phi_{0} / \mu$. The Reynolds number is $R e=\mathrm{U}_{0} \mathrm{~h} / v$, the Schmidt number is $\mathrm{Sc}=v / \mathrm{D}_{i}$ and the Peclet number is $\mathrm{Pe}=\operatorname{ReSc}$, where $\mu$ is the coefficient of viscosity and $v$ is the kinematic viscosity. 
The nondimensional steady state equations of motion for the ionized fluid with electroosmotic body forces are

$$
\begin{aligned}
\epsilon_{1} \frac{\partial u}{\partial x}+\frac{\partial v}{\partial y}+\epsilon_{2} \frac{\partial w}{\partial z}= & 0 \\
\operatorname{Re}\left(\epsilon_{1} u \frac{\partial u}{\partial x}+v \frac{\partial u}{\partial y}+\epsilon_{2} w \frac{\partial u}{\partial z}\right)= & -\epsilon_{1} \frac{\partial p}{\partial x}+\left(1-\frac{\epsilon_{1}}{\Lambda} \frac{\partial \phi}{\partial x}\right) \frac{\beta}{\epsilon^{2}}(g-f) \\
& +\nabla^{2} u \\
\operatorname{Re}\left(\epsilon_{1} u \frac{\partial v}{\partial x}+v \frac{\partial v}{\partial y}+\epsilon_{2} w \frac{\partial v}{\partial z}\right)= & -\frac{\partial p}{\partial y}-\frac{\partial \phi}{\partial y} \frac{\beta}{\Lambda \epsilon^{2}}(g-f)+\nabla^{2} v \\
\operatorname{Re}\left(\epsilon_{1} u \frac{\partial w}{\partial x}+v \frac{\partial w}{\partial y}+\epsilon_{2} w \frac{\partial w}{\partial z}\right)= & -\epsilon_{2} \frac{\partial p}{\partial z}-\frac{\partial \phi}{\partial z} \frac{\beta}{\Lambda \epsilon^{2}}(g-f)+\nabla^{2} w
\end{aligned}
$$

Here $\epsilon=\lambda / h, \beta=c / I, \Lambda=h E_{0} / \phi_{0}, I=\sum_{i} z_{i}^{2} c_{i}$ and $\lambda$ is the EDL thickness. The velocity field is coupled to the mass transfer equations and the equation for the potential. The nondimensional pressure is $p=p^{*} h / \mu U_{0}$ and

$$
\nabla^{2}=\frac{\partial^{2}}{\partial y^{2}}+\epsilon_{1}^{2} \frac{\partial^{2}}{\partial x^{2}}+\epsilon_{2}^{2} \frac{\partial^{2}}{\partial z^{2}}
$$

The width of the channel is of the order of the length of the channel, which is much larger than the height of the channel, so $\epsilon_{2}=\frac{h}{w} \ll 1$. Thus all gradients with respect to $z$ are neglected and the flow is treated as two-dimensional.

The wall mole fractions are obtained on the assumption that the average electrochemical potential in the channel is the same as that in the reservoir. Zheng et al. [9] described the procedure to obtain wall mole fractions from the electrochemical equilibrium requirements. Since the electrolyte consists of monovalent cation and monovalent anions (sodium and chlorine) the mole fraction sum is one at the boundary. The potential along the wall is set to zero, since only the difference in potential matters. The channel is assumed to be sufficiently long upstream and downstream of the wall mounted patches and the flow is assumed to be a fully developed EOF far upstream and downstream of the patches. The total channel length is $2 \mathrm{~L}$ with ends at $x= \pm \mathrm{L}$ and the 
patches centred about $x=0$. Thus the boundary conditions far upstream $(\mathrm{x}=-\mathrm{L})$ are

$$
u=u^{\text {in }}, \quad v=0, \quad \phi=\phi^{\text {in }}, \quad g=g^{\text {in }}, \quad f=f^{\text {in }}, \quad \frac{\partial p}{\partial x}=0 .
$$

Far downstream $(x=\mathrm{L})$ Neumann boundary conditions are applied:

$$
\frac{\partial u}{\partial x}=0, \quad \frac{\partial v}{\partial x}=0, \quad \frac{\partial \phi}{\partial x}=0, \quad \frac{\partial g}{\partial x}=0, \quad \frac{\partial f}{\partial x}=0, \quad \frac{\partial p}{\partial x}=0
$$

On the channel walls $(y=0$ and $y=1)$ but not about the patches,

$$
u=v=0, \quad \phi=0, \quad f=f^{0}, \quad g=g^{0} .
$$

The boundary conditions along the patches are

$$
u=v=0, \quad \phi=\phi_{p}, \quad f=f_{p}, \quad g=g_{p} .
$$

The values $u^{\text {in }}, \phi^{\text {in }}, g^{\text {in }}, f^{\text {in }}$ are for fully developed EOF. These are obtained by solving similar governing equations with the symmetry conditions.

The nondimensional overpotential along the patches is $\phi_{p}$ and the corresponding wall mole fractions are $f_{p}=f^{0} e^{\phi_{p}}$ and $g_{p}=g^{0} e^{-\phi_{p}}$, which satisfies the equilibrium condition of the electrochemical potential at $y=0$ and $y=1$. Thus the wall $\zeta$ potential is equal but opposite to the patch potential.

For computational purposes the mole fractions are scaled with the wall value $g^{0}$, that is, $\bar{g}=g / g^{0}, \bar{f}=f / g^{0}$. The governing equations are invariant to the rescaling of the mole fractions by the parameter $\delta^{2}=\epsilon^{2} / \beta g^{0}$. The governing nonlinear coupled set of PDEs are solved through a numerical scheme based on the SIMPLE (semi-implicit method for pressure-linked equations) algorithm. The Poisson equation for potential (Eq. (2)) is solved through a successive over-relaxation (SOR) technique. The algorithm was tested for accuracy by comparing with previously published results [11]. For validation, a comparison of the streamwise velocity is made with the lattice Boltzmann 
solution obtained from the Poisson-Boltzmann model (LPBM) for a channel height of $0.8 \mu \mathrm{m}$, as presented by Wang et al. [10]. The comparison is shown in Figure 2 for different values of the external electric field and $\zeta$ potential. Figure 2 shows excellent agreement with our computed solution based on the NP model. The difference between two results is $0.5 \%$ for $\mathrm{E}_{0}=500 \mathrm{~V} / \mathrm{m}$ and $0.1 \%$ for $\mathrm{E}_{0}=10^{3} \mathrm{~V} / \mathrm{m}$. Hence, at higher channel heights with larger external electric potential, the NP and PB models show very little difference.

\section{Results and discussions}

The EOF considered for the simulation process is a strong electrolyte case, where water is the solvent and $\mathrm{NaCl}$ is the electrolyte solute. For a strong electrolyte case, the molar concentration at the wall is $\mathrm{Na}^{+}=0.154 \mathrm{M}$, $\mathrm{Cl}^{-}=0.141 \mathrm{M}$ and for water, 55.6 M. These correspond to a EDL thickness of $0.8 \mathrm{~nm}$. Physical parameters of the medium (water) are the dielectric constant 78.54 , viscosity $\mu=10^{3} \mathrm{~kg} / \mathrm{ms}$ and density $\rho=1000 \mathrm{~kg} / \mathrm{m}^{3}$. The temperature of the solvent is $300 \mathrm{~K}$, and the external electric field is $10^{6} \mathrm{~V} / \mathrm{m}$. The overpotential (dimensionless) along the patches is $\phi_{\mathrm{p}}=0.2$, which corresponds to an EDL thickness of $0.78 \mathrm{~nm}$. Away from the patches, the flow is predominantly along the direction of the applied electric field which is similar to what is observed in the case of a plane nano channel. In this region (far away from the patches), the bulk fluid is electrically neutral $(h>10 \mathrm{~nm})$ and the flow is governed by the viscous effect, which leads to a constant bulk flow. The EDL which forms along the patches is different to the EDL which forms along the channel walls, resulting in a fluid flow rate difference.

For a pressure driven flow, flow separates from the channel wall when there is any wall nonhomogeneity for any value of the Reynolds number [4]. The mechanism of the EOF is different from that of a pressure driven flow. The adverse pressure gradient close to the patches, which is very high compare to the plane EOF, induces a flow separation. The streamlines for heterogeneous channel walls is presented in Figure 3(a). The nonhomogeneity along the 


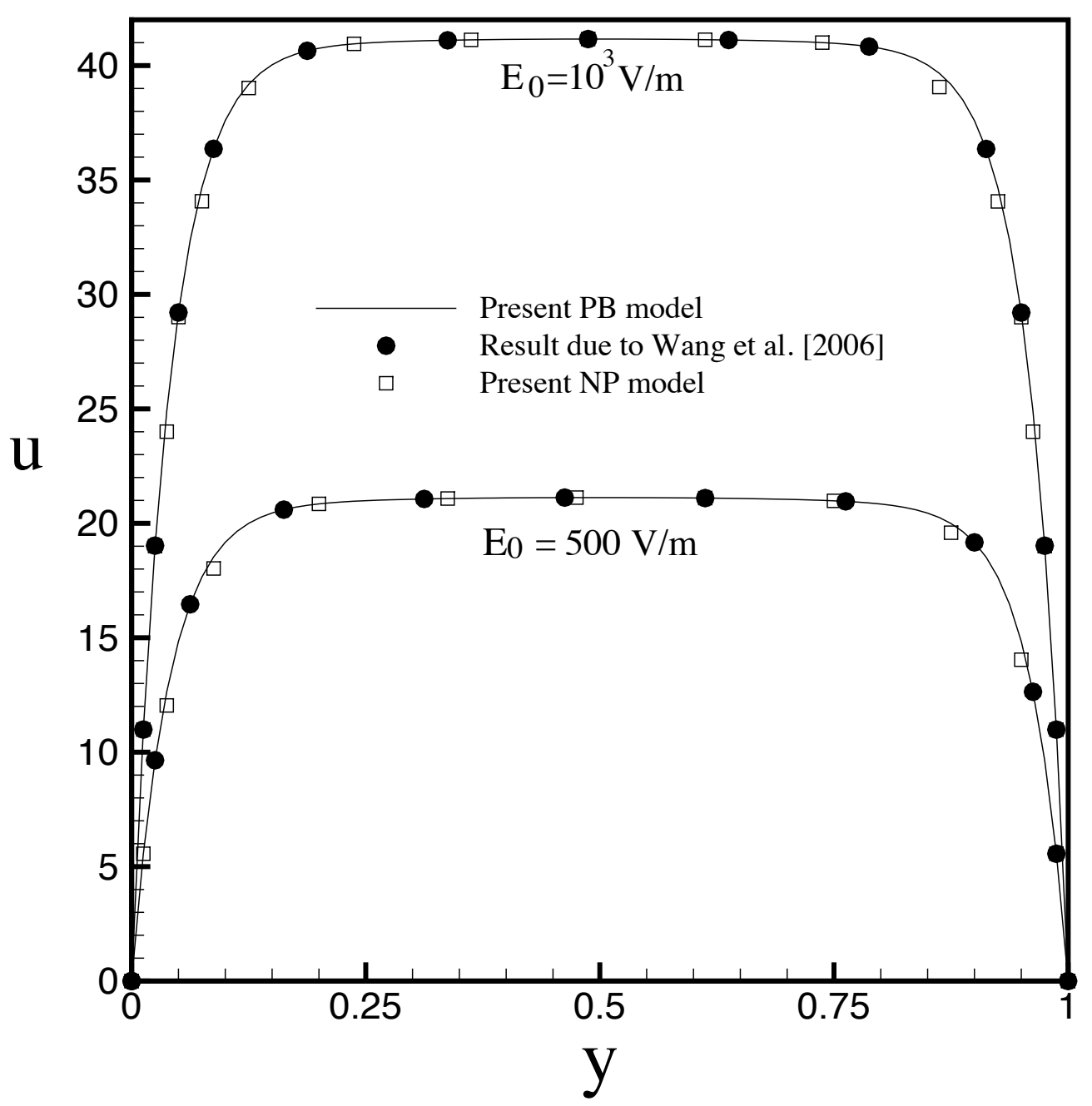

Figure 2: Comparison of the axial velocity profile for $\zeta=-50 \mathrm{mV}$. The EOF is considered in a homogeneous micro channel of height $0.8 \mu \mathrm{m}$ and the molar concentration of the ion is $10^{-4} \mathrm{M}$. The solid lines were obtained from the PB model; the open squares were obtained from the NP model; and the closed circles were obtained by Wang et al. [10]. 
channel walls is created by symmetrically arranged external patches with $\phi_{p}=0.2$. The dimensional potential outside the patches is set at $25 \mathrm{MeV}$ for a channel height of $50 \mathrm{~nm}$. The plug flow nature of the velocity, which is the classical form of the electroosmotic flow velocity profile, emerges far upstream and downstream of the patches. The velocity and the potential behaviour far upstream and downstream of the patches is due to the negligible convective effects. The streamlines for asymmetrically placed potential patches along the channel walls is presented in Figure 3(b). The streamlines show the presence of a negative vortex adjacent to the potential patch for both symmetric and asymmetric cases. The length of the vortex is reduced for asymmetrically placed potential patches, where the EDL strength is low.

The flow approaches an irrotational nature both upstream and downstream of the patches. The flow acceleration in the region above the vortex is maximum. The streamline patterns show that the fluid velocity is enhanced in the case of a asymmetric heterogeneity compare to symmetric heterogeneity. A comparison of the PB and NP model is presented in Figure 2. It shows that at larger channel heights the PB the NP model show very similar flow profiles.

The distribution of mole fractions for the symmetrically arranged patches, using the same physical parameters as above, are presented in Figure 4(a). This figure shows that the bulk fluid is electrically neutral, except near the patches. The surplus of positive ions ( $\mathrm{g}$ ) in the regions above the potential patches is evident. We find from the distribution of $g$ and $f$ that the convection has almost no effect on the molar concentration of ions in the core region. The distribution of mole fractions for asymmetrically arranged patches is presented in Figure 4(b). The mole fraction distribution along the heterogeneous region shows non-neutrality in the core region of channel, which may cause maximum mixing enhancement.

An important application of nonuniformity in the wall potential for EOF, particularly in biology, is the enhancement of mixing of different chemical species. The proposed mixing scheme is quantified by evaluating the mixing 


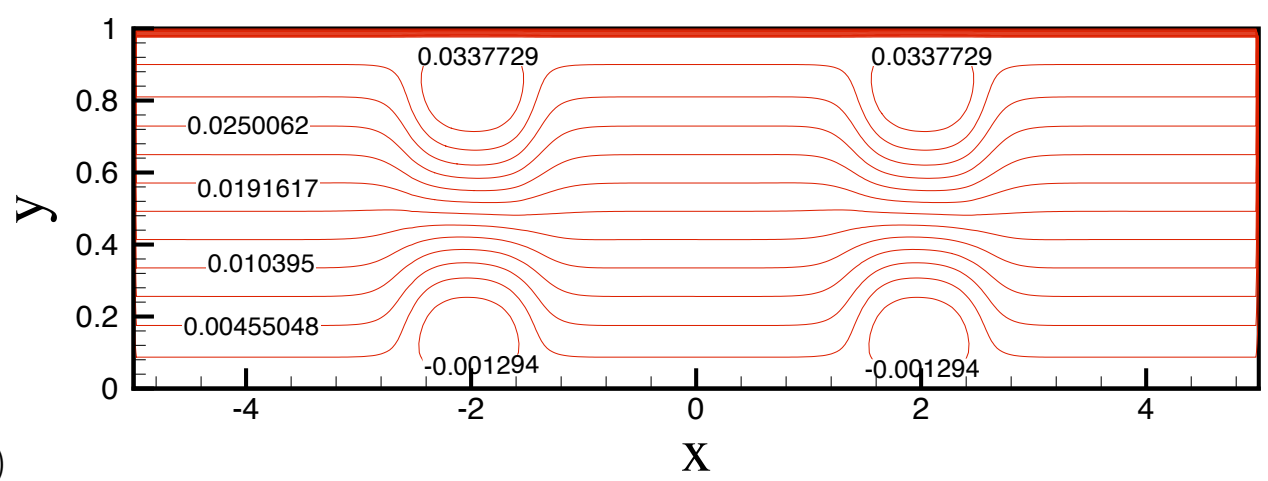

(a)

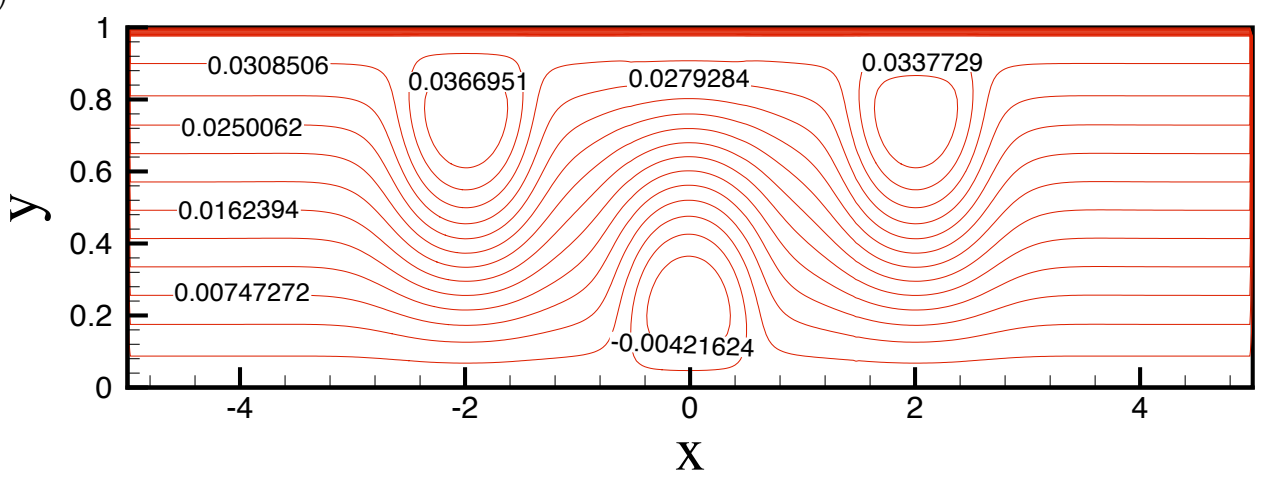

(b)

Figure 3: Streamlines for (a) symmetrically and (b) asymmetrically arranged patches, when the surface $\zeta$ potential is $-25 \mathrm{mV}$ for channel height $50 \mathrm{~nm}$.

efficiency [11],

$$
\sigma=\left[1-\frac{\int_{0}^{\mathrm{L}}\left|\mathrm{C}-\mathrm{C}_{\infty}\right|}{\int_{0}^{\mathrm{L}}\left|\mathrm{C}_{0}-\mathrm{C}_{\infty}\right|}\right] \times 100 \%,
$$

where $\mathrm{C}$ is the species concentration, $\mathrm{C}_{\infty}$ is the species concentration in the perfectly mixed condition (concentration for a fully developed flow after a steady state), and $\mathrm{C}_{0}$ is the species concentration in the completely unmixed condition (at the initial stage). Thus, a mixing efficiency $\sigma=100 \%$ indicates a perfectly mixed state, while a mixing efficiency $\sigma=0 \%$ indicates a completely unmixed state. The distribution of pressure along the $x$-axis for symmetrically 
(a)

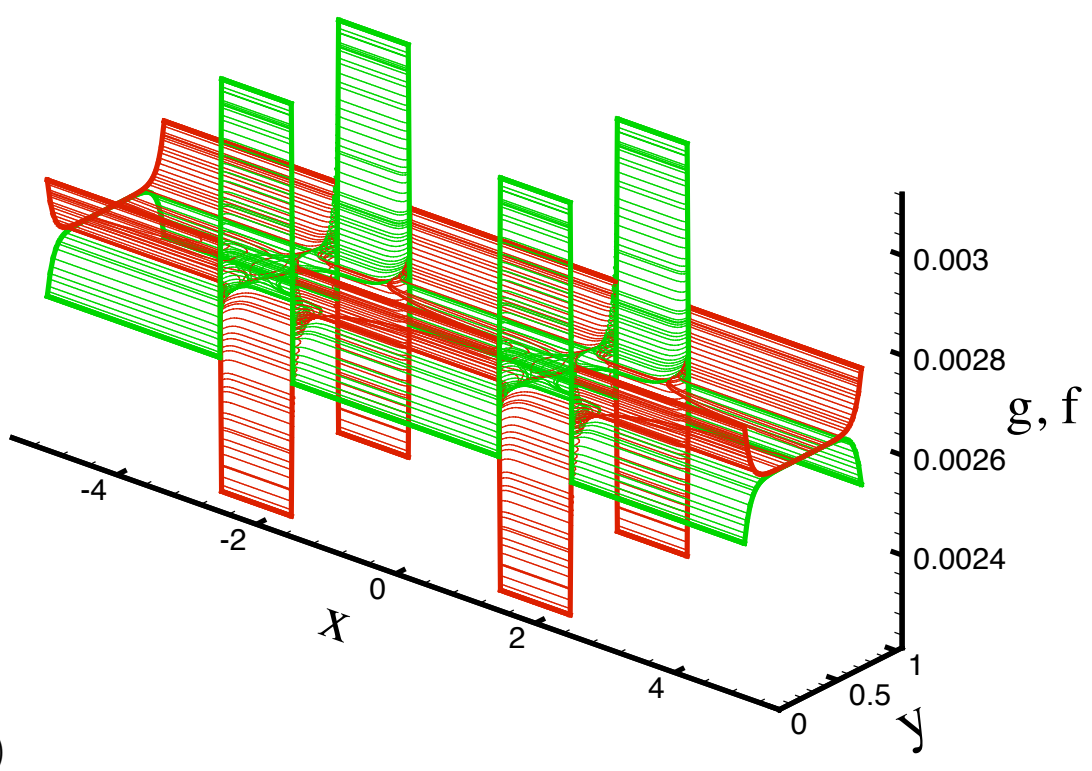

(b)

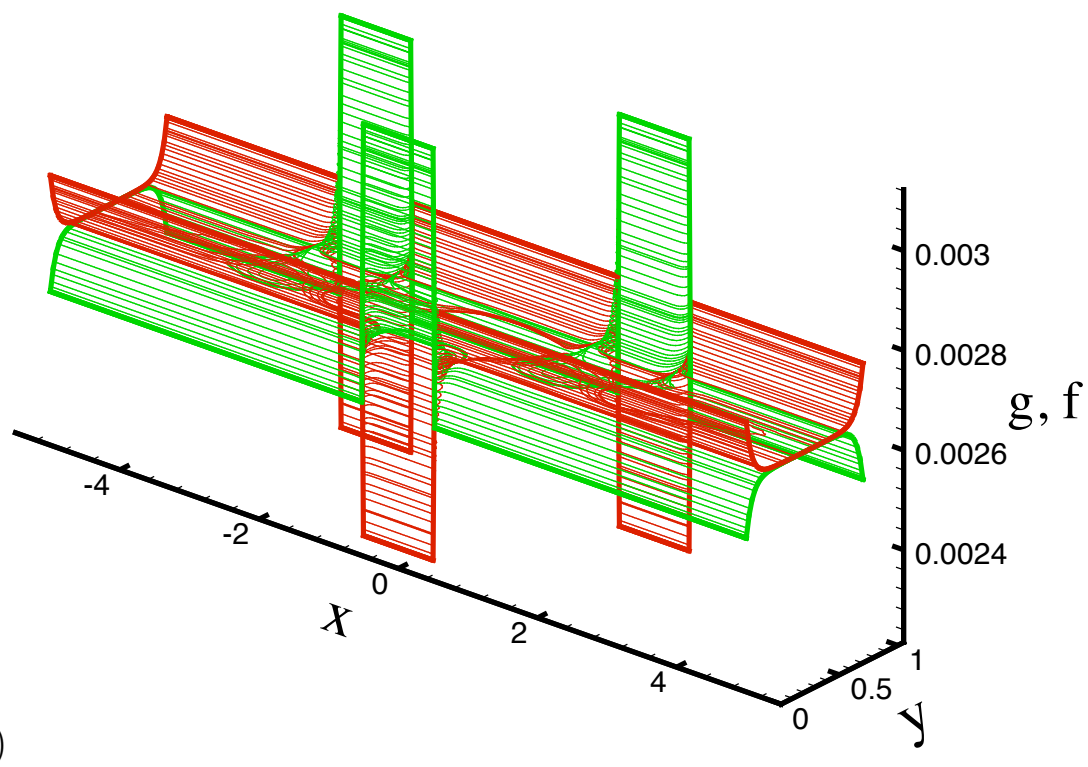

Figure 4: Distribution of mole fractions $g$ (red) and $f$ (green) for (a) symmetrically and (b) asymmetrically arranged paches with the same physical parameters used in Figure 3. 


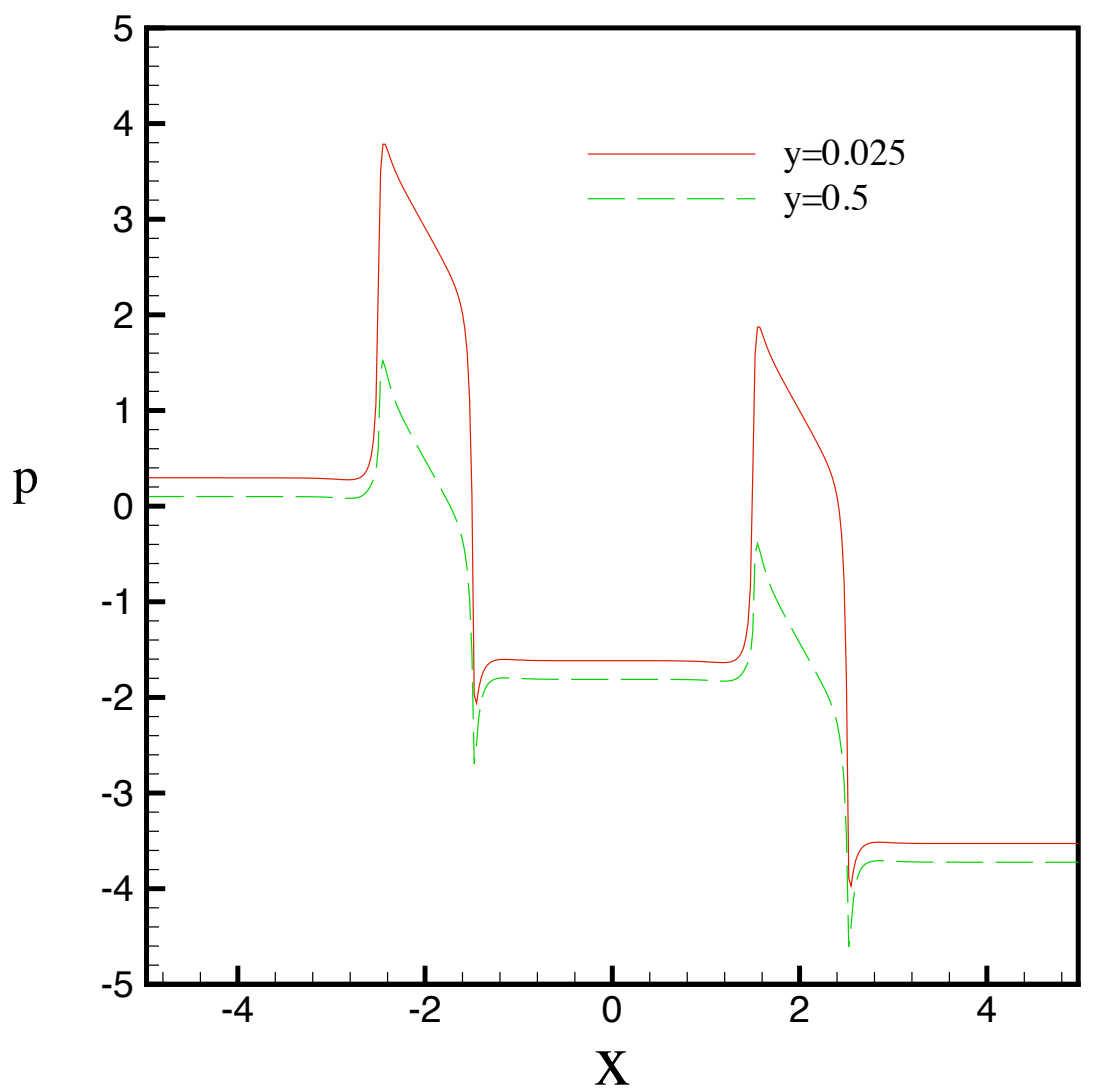

Figure 5: Distribution of pressure along $x$-axis, with the same physical parameters used in Figure 3.

arranged patches and for two different $y$ values is shown in Figure 5 when $\mathrm{h}=50 \mathrm{~nm}$ and $\phi_{\mathrm{p}}=0.2$ for the case of a strong solution. The distribution of pressure clearly shows that the pressure is high near the upstream side of the patch, and a strong high pressure gradient develops above the patches.

The mixing efficiency for both the symmetric and asymmetric cases with height $\mathrm{h}=50 \mathrm{~nm}$ and $\phi_{\mathrm{p}}=0.2$ is presented in Figure 6. The mixing performance is enhanced when the asymmetric condition is applied. For EOF in micro or 


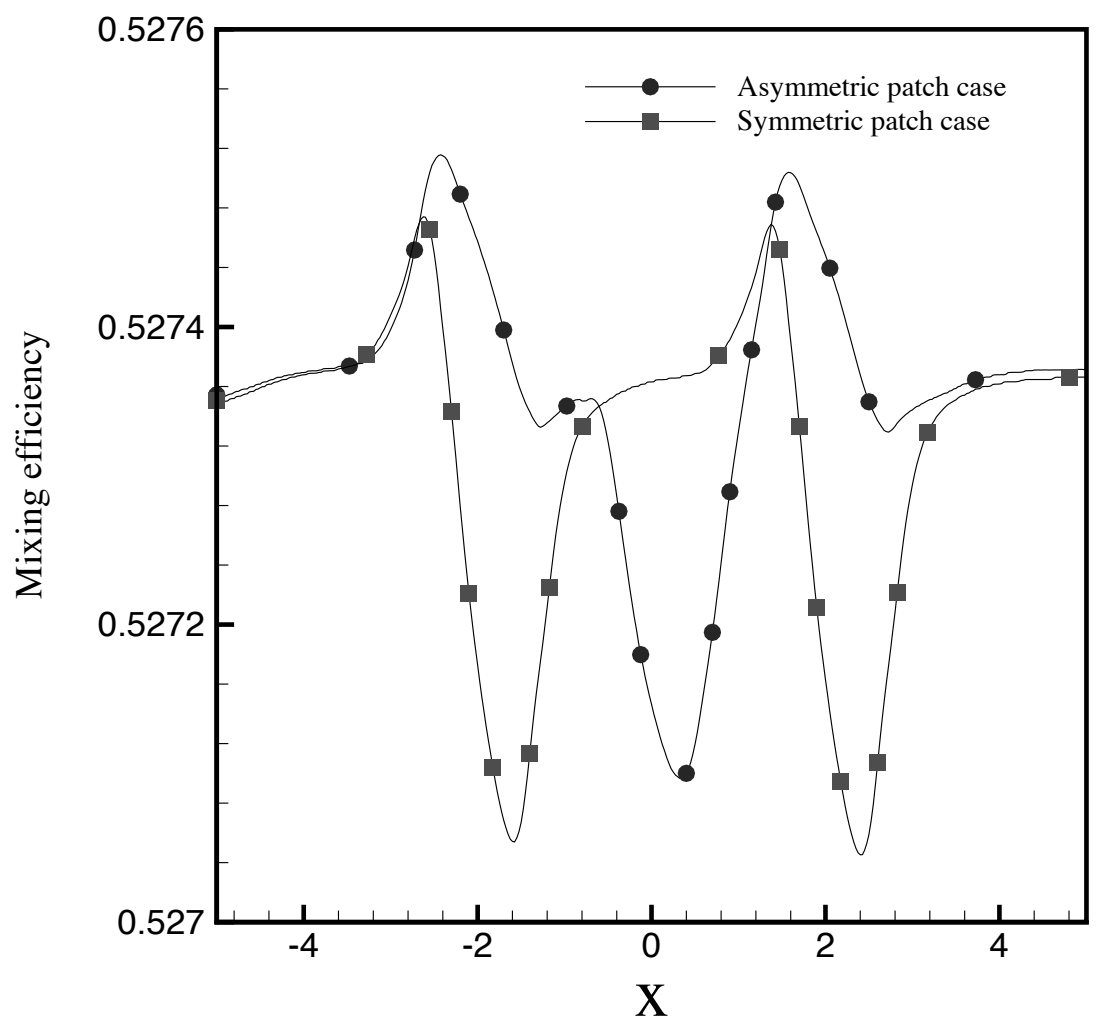

Figure 6: Mixing efficiency, for symmetric and asymmetric cases, with the same physical parameters used in Figure 3.

nano channels, various design parameters are also effective for the mixing of species. For long micro or nano channels, mixing is purely diffusive and hence the mixing efficiency is poor. The mixing efficiency of a straight channel is 0.5272 , and our results show that the introduction of potential patches significantly increases the mixing efficiency from $65 \%$ to $85 \%$. 


\section{References}

[1] F. Tian, B. Li and D. Y. Kwok, Tradeoff between mixing and transport for EOF in hetero. microchannels with nonuniform surface potentials. Langmuir 21:1126-1131, 2005. doi:10.1021/la048203e C48

[2] R. H. Liu, M. A. Stremler, K. V. Sharp, M. G. Olsen, J. G. Santiago, R. J. Adrian, H. Aref and D. J. Beebe, Passive mixing in a three dimensional serpentine microchannel. J. Microelectromech. S. 9:190-197, 2000. doi:10.1109/84.846699 C48

[3] Z. M. Wu, Z. J. Zhao, J. X. Yang, L. P. Liu and X. L. Yang, Freq.-modulation-type MI sensor with nanocrystalline ribbon core. Sensor. Actuat. A:Phys. 121:430-433, 2005. doi:10.1016/j.sna.2005.03.019 C48

[4] S. Bhattacharyya and A. K. Nayak, Combined Effect of Surface Roughness and Heterogeneity of Wall Potential on Electroosmosis in Microfluidic/Nanofuidic Channels. J. Fluids Eng. 132:041103, 2010. doi:10.1115/1.4001308 C48, C55

[5] A. Ajdari, Electro-Osmosis on Inhomogeneously Charged Surfaces. Phy. Rev. Lett. 75:755-758, 1995. doi:10.1103/PhysRevLett.75.755 C49

[6] D. Erickson and D. Li Influence of surface heterogeneity on electrokinetically driven microfluidic mixing. Langmuir 18:1883-1892, 2002. doi:10.1021/la015646z C49

[7] L. M. Fu, J. Y. Lin and R. J. Yang, Analysis of electroosmtoic flow with step change in zeta potential. J. Colloid. Interf. Sci. 258:266-275, 2003. doi:10.1016/S0021-9797(02)00078-4 C49

[8] H. Watzig, S. Kaupp and M. Graf, Inner surface properties of capillaries for electrophoresis. Trac-Trend. Anal. Chem. 22:588-604, 2003. doi:10.1016/S0165-9936(03)01102-6 C49 
[9] Z. Zheng and D. Hansford, \& A. T. Conlisk, Effect of multivalent ions on EOF in micro- and nanochannels. Electrophoresis 24:3006-3017, 2003. doi:10.1002/elps.200305561 C53

[10] J. Wang, M. Wang and Z. Li, Lattice Poisson-Boltzmann Simulations of Electro-Osmotic Flows in Microchannels. J. Colloid. Interf. Sci. 296:729-736, 2006. doi:10.1016/j.jcis.2005.09.042 C55, C56

[11] A. K. Nayak, An Analysis of Steady/Unsteady Electroosmotic flows Through Charged cylindrical nano channel. Theor. Comp. Fluid Dyn. 27:885-902, 2013. doi:10.1007/s00162-013-0295-0 C54, C58

\section{Author address}

1. A. K. Nayak, Department of Mathematics, Indian Institute of Technology Roorkee, Roorkee 247667, India.

mailto:nayakfma@iitr.ac.in 